\title{
Aplicaciones de la musicoterapia en educación especial y en los hospitales
}

\author{
Elena Ortega, Laura Esteban, Angeles F. Estévez y Diego Alonso \\ Universidad de Almería (España)
}

En este trabajo se hace una amplia revisión de aquellos estudios experimentales relacionados con la efectividad de la utilización de la musicoterapia, tanto en un contexto de educación especial como en los hospitales. La mayor parte de los estudios revisados aportan pruebas a favor de la hipótesis de que la utilización de la musicoterapia en niños con necesidades educativas especiales tiene efectos positivos en distintos aspectos, como son: reducción de la ansiedad, mejora del desarrollo emocional, mayor equilibrio psicofísico y emocional, y aumento del nivel de comunicación e interacción social. En un contexto hospitalario, la musicoterapia se ha mostrado eficaz principalmente como forma de reducir la ansiedad, y parcialmente, para calmar el dolor, aunque en este último aspecto, los datos son contradictorios. Desde un punto de vista metodológico, los estudios revisados adolecen de serios problemas, necesitándose, por este motivo, un mayor rigor experimental en los estudios que se realicen en este campo. Mientras tanto, no se podrán enunciar resultados concluyentes sobre la efectividad de la música como herramienta terapéutica en estos contextos.

Palabras clave: Musicoterapia, efectos musicoterapia, educación especial, ansiedad.

Applications of music therapy in special education and in hospitals. In this paper we present a wide review of those experimental studies related to the efficiency of the utilization of the music therapy both in a context of special education and in the hospitals. Most of the checked studies contributes evidence in favour of the hypothesis of the utilization of the music therapy in children with learning disabilities has positive effects in different aspects, as they are: reduction of the anxiety, improvement of the emotional development, bigger psycophysical and emotional balance, and increase of the level of communication and social interaction. In a hospitable context, the music therapy has proved to be effective principally as a tool to reduce the anxiety and, partially, to calm the pain, though in this aspect the information is contradictory. From a methodological point of view, the checked studies suffer from serious problems, being needed, for this motive, a bigger experimental rigor in the studies that are carried out in this field. Meanwhile, conclusive results will not be able to be enunciated on the efficiency of the music as therapeutic tool in these contexts.

Key words: Music therapy, therapeutic effects of music, learning disabilities, anxiety.

Correspondencia: Diego Alonso. Departamento de Neurociencia y CC. de la Salud. Facultad de Psicología. Universidad de Almería. La Cañada de San Urbano s/n, C.P. 04120. Almería (España).E-mail: dalonso@ual.es 
El impacto de la música sobre el comportamiento ha sido discutido y documentado a través de la historia. Las referencias a la universalidad de la música, magia y mitos confirman la creencia de que ésta tiene una potente influencia en el ser humano. Pese a este reconocimiento histórico sobre los efectos de la música, no es hasta la década de los 50 cuando se comienza a desarrollar la musicoterapia o terapia musical. De hecho, una de las primeras definiciones de musicoterapia parte del profesor Thayer Gaston $(1957,1968)$ quien, en los años 50, la define de la siguiente forma: "Música es la ciencia o el arte de reunir o ejecutar combinaciones inteligibles de sonidos en forma organizada y estructurada con una gama de infinita variedad de expresión, dependiendo de la relación de sus diversos factores componentes (ritmo, melodía, volumen y cualidad tonal). Terapia tiene que ver con "cómo" puede ser utilizada la música para provocar cambios en las personas que la escuchan o la ejecutan". Las dos ideas centrales que conforman esta primera definición de musicoterapia son, pues, el uso de la música y los cambios que se generan (Poch, 2002). Así, dicha definición, dada por el primer profesor universitario de musicoterapia, deja patente que el uso adecuado de la música, teniendo en cuenta sus diferentes componentes y adecuándolos a los pacientes, provocará cambios beneficiosos en las personas.

Recientemente, Hillecke, Níkel y Bolay (2005) exploran los factores que hacen que la musicoterapia sea efectiva, destacando los siguientes: (1) factor atencional, la música es un estímulo auditivo capaz de captar la atención mucho mejor que otros estímulos sensoriales, y de generar distracción y relajación; (2) factor emocional, la música puede servir para modular las emociones, ya sea porque una melodía activa directamente determinadas emociones o porque activa el recuerdo de emociones asociadas; (3) factor cognitivo, la comprensión de la música implica el pensamiento y la creación de una experiencia subjetiva, lo que puede ayudar a cambiar determinadas cogniciones y significados subjetivos previamente establecidos; (4) factor conductual, la música es una herramienta capaz de activar el movimiento, ya que está estrechamente relacionada con la danza (así, se puede utilizar en la mejora de los problemas motores asociados con diferentes trastornos); y, (5) factor de comunicación, la música constituye una forma de comunicación no verbal de gran ayuda en el tratamiento de los problemas relacionados con la interacción entre personas, resultando especialmente efectiva como vehículo de expresión de emociones en las personas que no pueden comunicarse de forma verbal (Juslin y Sloboda, 2001).

Con respecto a la aplicación de la musicoterapia, podemos decir que en su origen estaba limitada al tratamiento de los trastornos de comunicación en niños y adolescentes (v.g., Alvin, 1978; Nordoff y Robbins, 1977). Sin embargo, actualmente el ámbito de aplicación de la misma es mucho más amplio. Concretamente, según Lacarcel (1990), se puede aplicar: (1) a nivel grupal, como técnica lúdica y de 
relajación, según las necesidades, objetivos y expectativas del grupo al que va dirigida; (2) como terapia a nivel de psicopatología en centros psiquiátricos; (3) en las terapias interpersonales -parejas, familia, grupos especiales, etc.-; (4) como terapia de apoyo en el tratamientos del alcoholismo y la drogodependencia; (5) en tratamientos psicomotores de diferentes discapacidades o deficiencias; (6) en niños y adolescentes con problemas de conducta e inadaptación social; y, (7) para superar bloqueos de la comunicación -timidez, inhibición, mutismo, dificultad de expresión, etc.-. Por otra parte, en cuanto a la población que puede beneficiarse del uso de la musicoterapia, la American Music Therapy Association (NAMT, citado en Poch, 2002) señala que la terapia musical parece ser útil con quienes presentan (en orden de frecuencia): retraso en el desarrollo; desórdenes en el comportamiento; desórdenes afectivos; problemas psicológicos; población general en edad escolar; discapacitados múltiples; problemas de lenguaje; autistas; problemas visuales; déficit neurológicos; problemas auditivos (sordos e hipoacúsicos); abuso de sustancias; abusos sexuales; prematuridad; y, daño cerebral. Así mismo, se pueden incluir niños con síndrome de Rett o de Williams, personas con desórdenes alimenticios, niños con espina bífida o problemas de espalda, ancianos, pacientes con demencia, etc.

Dado el amplio abanico de aplicaciones de la musicoterapia, cuyo tratamiento en profundidad podría ser objeto de un manual dedicado exclusivamente a este fin, en el presente trabajo nos vamos a centrar en sólo dos de sus posibles ámbitos de aplicación, uno que goza de una amplia trayectoria, el de la educación especial, y otro de reciente surgimiento pero con un gran futuro: la aplicación de la terapia musical en los hospitales.

\section{MUSICOTERAPIA EN EDUCACIÓN ESPECIAL}

Mediante la aplicación de la musicoterapia se ha podido constatar que muchos niños han mejorado considerablemente su deficiencia (Ruiz, 2005). Concretamente, los niños con necesidades educativas especiales son remitidos con frecuencia a terapia musical para enfrentarse y mejorar sus necesidades de comunicación, cognitivas, sensorio-motores o perceptivo-motoras, sociales, emocionales y psicológicas. Los terapeutas musicales trabajan para mejorar sus destrezas, cambiar comportamientos específicos o enseñarles nuevas habilidades a través de la experiencia musical.

En términos generales, la acción beneficiosa que la música ha ejercido sobre los niños con discapacidades, se podría concretar de la siguiente manera (Lacarcel, 1990): (1) la música como medio de expresión y de comunicación favorece el desarrollo emocional, mejora de un modo considerable las percepciones y la motricidad del niño, así como su afectividad; (2) favorece la manifestación de tensiones, 
problemas, inquietudes, miedos, bloqueos, etc., disminuyendo de este modo la ansiedad; (3) se consigue un mayor equilibrio psicofísico y emocional; (4) los estímulos rítmicos aumentan el rendimiento corporal así como el riego sanguíneo cerebral; y, (5) en niños con grandes dificultades de comunicación se ha comprobado que responden al estímulo musical más que a cualquier otra clase de estímulo.

A continuación se van a presentar algunas de las dificultades más comunes en educación especial y los efectos que sobre éstas tiene la musicoterapia.

\section{Retraso en el desarrollo y trastornos de aprendizaje}

En este apartado se incluyen desórdenes que tienen su origen durante la infancia y que continúan indefinidamente, afectando a diversas capacidades. Un ejemplo de retraso en el desarrollo lo constituye el retraso mental. Son diversos los estudios en los que se ha mostrado que la utilización de la musicoterapia tiene efectos beneficiosos en niños (Hanks, 1986; Knill, 1983; Preza, Baboci, Ashta y Lleshi, 1990; Wesecky, 1986; Zappella, 1986) así como en adultos con retraso mental (Bolton y Adams, 1983; Oldfield y Adams, 1990), lo que no es de extrañar teniendo en cuenta que los diferentes elementos musicales (ritmo, melodía, timbre, textura, tempo) implicados en actividades tan diferentes como escuchar, cantar, jugar e improvisar música pueden influir y producir cambios en los componentes fisiológicos, psicológicos y emocionales del patrón de crecimiento normal de un niño (Taylor, 1990). Con este tipo de niños la musicoterapia intenta crear un ambiente alegre y divertido, que les permita aprender mediante la consecución de logros o éxitos y no de fracasos. Mientras aprenden una simple canción o tocan un instrumento, simultáneamente están mejorando su contacto ocular, capacidad atencional, seguimiento de instrucciones, imitación verbal, memoria, psicomotricidad fina y discriminación auditiva. Además, uniendo palabras con tonos y frases con melodías, los terapeutas consiguen mejorar la comunicación de estos niños (Cohen y Masse, 1993; Popovici, 1995; Rejto, 1973; Seybold, 1971).

La música les ofrece, a su vez, oportunidades para aprender comportamientos sociales y motores adecuados, incrementando su interacción social mediante el trabajo en grupo, lo que redunda en una autoestima más positiva. La musicoterapia en niños con retraso persigue una adecuada adaptación al medio, mejorando de este modo las relaciones interpersonales y la comunicación, lo que tiene como consecuencia una mayor autoestima y confianza en sí mismo. Mediante esta terapia, se estimula también su memoria, atención, reflexión $\mathrm{y}$, en general, sus facultades intelectuales, así como las psicomotoras (coordinación, esquema corporal, lateralidad, percepción espacial y temporal, etc.). Existen numerosos estudios que demuestran el gran potencial de desarrollo de los niños con retraso, gracias a la aplicación exitosa de la musicoterapia (DiGiammarino, 1990; Dorow y Horton, 1982; 
Madsen, 1981). Incluso en el trastorno generalizado del desarrollo, la terapia musical realza habilidades funcionales, al tiempo que enriquece las capacidades creativas y expresivas.

Por otro lado, cabe destacar que los niños autistas, quienes, por lo general, rechazan las interacciones sociales, pueden llegar a realizar intentos comunicativos con los terapeutas a través de las diversas experiencias musicales a las que son expuestos. Como señalan Accordino, Comer y Heller (2007) en una reciente revisión sobre el tema, la gran aceptación de la musicoterapia en relación con el autismo ha coincidido con el hecho de que diversas investigaciones demuestran la capacidad musical de los autistas (Applebaum, Egel, Koegel e Imhoff, 1979; Bonnel et al., 2003; Foxton et al., 2003; Heaton, 2003, 2004; Heaton, Hermelin y Pring, 1998; Heaton, Pring y Hermelin, 2001; Mottron, Peretz y Menard, 2000). Sin embargo, según estos autores, existen pocos datos empíricos que avalen la utilización de la terapia musical en esta población ya que la mayor parte de la literatura disponible en este campo se limita a la descripción de un único caso. A pesar de esta limitación y de la necesidad de realizar estudios experimentales, Accordino y colaboradores destacan la gran variedad de técnicas utilizadas en los estudios revisados, entre las que se incluyen: entrenamiento del ritmo, sincronización musical, musicoterapia receptiva (escuchar música en vivo o grabada), musicoterapia activa (se utilizan diferentes juegos musicales) y musicoterapia de improvisación (se guía al paciente para que éste cree música de forma espontánea).

Por su parte, mediante la musicoterapia, el niño con parálisis cerebral puede desarrollar sus capacidades residuales con eficacia terapéutica. Para Benenzon (1981) "los sectores indemnes del cerebro poseen reservas de las que el organismo puede extraer elementos de sustitución, compensación o restitución de los defectos resultantes del daño sufrido. Es esa reserva la que el musicoterapeuta ayudará a desarrollar, sobre todo como apertura". Con una práctica adaptada a cada necesidad, el niño con parálisis cerebral se irá liberando progresivamente de la tensión y ansiedad que le produce su limitación. La enseñanza del control del cuerpo a través del ritmo, instrumentos, voz y canto, podrá facilitar la organización de los movimientos para que el dominio del cuerpo sea regido por el cerebro. De hecho, la música proporciona -por sus connotaciones de tiempo y espacio- sensación de movimiento. El trabajo más importante con este tipo de niños se realiza, así pues, con su cuerpo, lo que va a proporcionarle desinhibición y autoestima al observar que es capaz de expresarse con sus propios medios mediante un diálogo espontáneo y variado. De esta manera, la musicoterapia puede proporcionar al niño con parálisis cerebral un medio para realizarse, ya que las limitaciones producidas por esta dolencia hacen que se sienta frustrado emocional y físicamente, aunque posea una inteligencia normal. La música le va a abrir nuevas formas de expresión y comunicación. Además, dado que los trastornos del lenguaje son comunes en paralíticos cerebrales, la musicoterapia les va a 
ayudar, no sólo a desarrollar el lenguaje, sino también a controlar y fortalecer los músculos oro-faríngeos.

Para finalizar, señalar que en dos estudios recientes se ha utilizado la musicoterapia en niños con déficit severos de aprendizaje, con la particularidad de que en ambos las sesiones de terapia musical se integran en el programa escolar. Coleman (2002) realiza el estudio en una escuela pública, mientras que Gladfelter (2002) asiste a una privada. En el segundo estudio, las sesiones, de cuarenta y cinco minutos de duración, se reparten a lo largo del curso escolar con una frecuencia semanal y consisten en la realización de actividades como tocar instrumentos musicales, cantar, escuchar música, crear canciones, improvisar o producir vídeos musicales. Con estas tareas se pretendía, entre otras cosas, incrementar la atención, desarrollar y mejorar las habilidades de procesamiento auditivo, mejorar las capacidades lingüísticas, desarrollar habilidades sociales adecuadas, fomentar la auto-expresión y mejorar las habilidades motoras finas y gruesas. Los resultados en ambos casos fueron positivos, lo que les lleva a señalar que la musicoterapia es una herramienta especialmente beneficiosa para los niños con necesidades educativas específicas ya que puede proporcionar nuevas vías para el aprendizaje y el desarrollo de los mismos. Igualmente, Sausser y Waller (2006) creen que la musicoterapia podría ayudar a los niños con trastornos emocionales y conductuales a superar algunos de los problemas que presentan, de un modo creativo y divertido. Así, la terapia musical permite que los niños con necesidades educativas especiales interaccionen positivamente con otros compañeros que están en clases ordinarias, al tiempo que les permite aprender juntos (Gunsberg, 1988; Hughes, Robbins, McKenzie y Robb, 1990; Humpal, 1991).

\section{Trastornos psicomotores}

Este tipo de niños puede tener problemas en el ámbito del desarrollo motor o dificultades en la coordinación motora, fina o gruesa. En todos los casos, el ritmo va a ayudar a desarrollar el control motor elemental y la coordinación sensoriomotora. Según Lacarcel (1990), mediante la utilización de ejercicios rítmicos podemos estimular y desarrollar la adquisición de destrezas sensoriomotrices de agilidad, equilibro y coordinación corporal; desarrollar un adecuado tono muscular para poder controlar un movimiento rítmico y armónico del cuerpo; descubrir el espacio, explorar y vivenciar el propio movimiento para tomar conciencia del lugar que ocupa el cuerpo en el espacio; conseguir mediante la orientación espacial el descubrimiento de la lateralidad; reeducar las coordinaciones y el sentido rítmico, como premisa para el aprendizaje de la lecto-escritura; etc. Por otra parte, hay que destacar que la práctica con instrumentos musicales puede ayudarles a mejorar su coordinación óculo-motriz, pero si éstos, además, son de viento, pueden controlar su respiración. Concretamente, para la terapia y reeducación visomanual se han de utilizar ritmos, audiciones 
musicales y canciones que impliquen la manipulación de instrumentos, percusiones corporales, percusiones sobre objetos, etc. Así mismo, el baile o la danza resultan muy útiles, especialmente cuando se integran varias partes del cuerpo en un mismo movimiento. Tocando en el piano melodías progresivamente más complejas, primero con una mano y después con ambas, se puede desarrollar también la coordinación. Por último, copiando letras de canciones se realizan los mismos movimientos oculares que son necesarios para leer palabras.

Con respecto al papel de la musicoterapia como herramienta de ayuda para el aprendizaje y la rehabilitación motora, Thau (1988) señala que la utilización de un ritmo externo (por ejemplo, el sonido de un metrónomo) ayuda a mejorar la coordinación neuromuscular, mientras que Yasuhara y Sugiyama (2001) encuentran que la terapia musical disminuye los movimientos estereotipados y mejora el movimiento en general de un grupo de niños con síndrome de Rett.

\section{Dificultades de comunicación}

Los niños con problemas de comunicación se pueden beneficiar de la musicoterapia de muchas maneras. Cantar ayuda a mejorar el habla y el lenguaje, y, de un modo más específico, resulta beneficioso para la memoria auditiva y para la fluidez verbal. Por su parte, los niños que no hablan son buenos candidatos para la terapia musical ya que, dado que carecen del significado explícito de la comunicación que usamos la mayoría, necesitan aprender otros métodos para poder expresarse.

Salvo que los problemas de lenguaje sean secundarios a otros trastornos, la mayoría de los niños que presentan dificultades para aprender a hablar y escribir suelen tener el resto de capacidades intactas. A pesar de ello, los niños con problemas de lenguaje se encuentran en desventaja, pues éste es el vehículo más importante de comunicación, expresión, desarrollo y aprendizaje. La musicoterapia va a tener una influencia positiva en la reeducación y tratamiento especial de estos niños ya que constituye un medio de rehabilitación que incide tanto desde el punto de vista puramente fisiológico, como puede ser la ejercitación de la voz, movimiento, etc., como desde la perspectiva emocional e intelectual que proporciona un desarrollo afectivo y cognitivo.

De un modo más concreto, desde el ámbito de la terapia musical se trabaja con niños que presentan afasia, agnosia auditiva, apraxia, dislalia, dislexia, disprosodia, disgrafía, disartria, ecolalia, tartamudez, y paladar y labio hendido (Lacarcel, 1990). En este sentido, la musicoterapia persigue una correcta articulación, puesto que los problemas que presentan estos niños de sustituciones, omisiones, adiciones, distorsiones de sonidos hablados, etc., podrían ser superados. Así mismo, pretende que los niños adquieran una adecuada vivencia del tempo y el ritmo, ya que las dificultades de tiempo de emisión de las palabras y de un ritmo y acentuación correctos, son 
problemas usuales en algunas deficiencias del habla. Por último, la terapia musical trabaja educando la voz, ya que estos niños suelen presentar anormalidades en la altura, intensidad o cualidad de la misma.

\section{Déficit de atención e hiperactividad}

Todo parece indicar que componer música, especialmente en grupos con compañeros de la misma edad, demanda una considerable atención y autocontrol, habiendo un acuerdo general de que la utilización y la práctica con tareas rítmicas conduce a una mejora en la organización interna y en el control de las conductas denominadas impulsivas (Gibbons, 1983; Thaut, 1992). Teniendo en cuenta estos datos, así como el hecho de que en algunos estudios se han utilizado tareas rítmicas para mejorar la conciencia corporal (Wigram, Pederson y Bond, 2002) y la coordinación motora (Gibbons, 1983; Howell, Flowers y Wheaton, 1995; Moore y Mathenius, 1987; Thaut, 1985), Rickson (2006) realiza un estudio con 13 niños diagnosticados con trastorno por déficit de atención con hiperactividad (TDAH). En dicho estudio se evaluaron dos condiciones, una en la que los participantes tocaban instrumentos de percusión siguiendo un patrón rítmico concreto propuesto por el terapeuta, y otro en el que se tocaban los mismos instrumentos pero de forma libre, improvisada. En ambos casos se observó una reducción de los síntomas asociados con la hiperactividad mejorando la ejecución de los niños en una tarea de repetición de patrones rítmicos generados por el ordenador. Además, se encontró que ambos grupos mejoraron, en general, sus habilidades para escuchar, atender e implicarse en actividades grupales, mostrando durante las sesiones de musicoterapia unos mayores niveles de atención sostenida, concentración y autocontrol que en otros tipos de tareas.

\section{Déficit sensoriales}

Tal y como señala Lacarcel (1990), en quien nos vamos a basar para desarrollar el presente apartado, bajo este término se incluyen niños con problemas de visión, audición o ambos. Con respecto a los discapacitados auditivos, debemos comenzar señalado que los niños con dificultades auditivas sufren diferentes grados que van, desde los que son completamente sordos, a los hipoacúsicos más o menos graves. La musicoterapia, como técnica y como expresión, aporta al niño hipoacúsico la vivencia de la música, e incluso aquellos que son al cien por cien sordos tienen posibilidades de reeducación a través de la misma, siempre teniendo en cuenta que el deficiente auditivo no "oye" la música como lo haría una persona normal, sino que percibe vibraciones y sensaciones acústicas sin una significación ni estimación concretas.

Actualmente se sabe que si el niño con déficit auditivo no es atendido adecuadamente durante su vida escolar, tiende a centrarse cada vez más en sí mismo. 
Esto origina problemas de índole afectiva, nerviosa y de integración social, ya que se siente limitado al no poder establecer una buena comunicación con sus iguales. La musicoterapia aporta al niño hipoacúsico la vivencia de la música mediante un método activo en el que queda implicado tanto a nivel individual como grupal. Por su parte, los niños sordos son capaces de responder al ritmo, de discriminar y comparar sonidos. Así, mediante el ritmo, el movimiento, juegos musicales, etc., se puede crear una variada y rica fuente de estímulos que le ayuden a desarrollar las percepciones auditivas, táctiles, kinestésicas y visuales. Podemos decir que el desarrollo rítmico en el niño sordo e hipoacúsico se puede alcanzar desde: el ritmo en las palabras (prosodias, canto, recitaciones, expresiones verbales, rimas, melodías, etc.) y el ritmo en el movimiento (percusiones corporales, marchas, danzas, expresión corporal, manejo de instrumentos...).

En resumen, la musicoterapia en disminuidos auditivos ayuda en: (1) su desarrollo cognitivo; (2) su autoestima, que se ve incrementada al sentirse capaces de hacer música y participar en actividades con sus compañeros; (3) el desarrollo de mejores relaciones interpersonales ya que el trabajo rítmico en grupo ayuda a sentirse integrados escolar y socialmente, al tiempo que ayuda a construir su propio yo y a adquirir confianza en sí mismos; (4) la obtención de un gran conocimiento del mundo que les rodea; (5) su discriminación auditiva, lo que les permite mejorar, a su vez, la locución; y, (6) el movimiento, procurando mejorar la coordinación motora y corporal, el equilibrio y el control postural.

Con respecto a los deficientes visuales, cabe señalar que aunque el niño ciego sigue un proceso de maduración igual al de los demás niños de su edad, presentando la misma necesidad de afecto y autoestima, no puede aprender de la misma manera que el resto de niños, sino que requiere de medios y métodos especiales que le han de ser enseñados desde los primeros momentos de su desarrollo. Aunque sus órganos de audición y tacto son normales, se van a sensibilizar mucho más para compensar, de esta manera, la carencia de visión, especialmente el oído, que será más precoz en esta función.

El niño con dificultades visuales responde fácilmente a la música gracias a la captación y memoria del sonido. Su interés y atención son excepcionales, ya que la música es una de las actividades de las que puede disfrutar plenamente, satisfaciendo de este modo necesidades de tipo emocional, intelectual y social. Así, la musicoterapia proporciona a estos niños grandes beneficios; entre otros, les permite recuperar su autoestima y sentirse autorrealizados, mejorar la comunicación y la integración social que tanto necesitan, les ayuda a expresar sus sentimientos, a desarrollar la creatividad, fantasía e improvisación, les facilita los movimientos, marcha, coordinación, etc. 


\section{MUSICOTERAPIA EN LOS HOSPITALES: TERAPIA E INVESTIGACIÓN}

En este apartado queremos comprobar si existen datos científicos que corroboren la idea generalizada de que la musicoterapia mejora la calidad de vida y reduce alguno de los síntomas físicos que muestran los pacientes hospitalizados. Basándonos en las investigaciones publicadas hasta el momento, estudiaremos cómo afecta la musicoterapia a pacientes con cáncer, a enfermos de corazón y a la ansiedad que se genera antes de una operación.

\section{Musicoterapia y cáncer}

La musicoterapia es una profesión en auge, dentro de la cual se encuadran diferentes prácticas y modelos teóricos. Entre los nuevos campos de actuación, se encuentra la oncología. Según Kruse (2003), en Estados Unidos, la mayoría de los musicoterapeutas que trabajan en oncología desarrollan su actividad profesional en este subcampo desde hace relativamente poco tiempo. Igualmente sucede en Reino Unido, donde tradicionalmente los musicoterapeutas habían centrado su trabajo en pacientes psiquiátricos y en trastornos del aprendizaje (Hanser, 2005).

La incorporación de la musicoterapia en el campo de la oncología no se limita a Estados Unidos y a Reino Unido, sino que se ha expandido por todo el mundo como podemos comprobar en el artículo de Aldridge (2003). Este autor realiza una revisión bibliográfica sobre el tema en cuestión, y cita datos procedentes de trabajos realizados en Australia, Canadá, China, Europa, Israel, Japón, Nueva Zelanda y Reino Unido.

La musicoterapia en oncología se centra en las necesidades psicológicas y físicas surgidas de los diferentes medicamentos y tratamientos utilizados actualmente (Aldridge, 2003). El punto de partida es la aceptación de que algunos tipos de cáncer no sólo implican dolores crónicos, sino que pueden representar serios cambios en la identidad del sujeto, momento en el que la persona necesita de sus recursos físicos y psicológicos (Aldridge, 2003). De hecho, diversos estudios muestran cómo pacientes que recurren a terapias alternativas (entre ellas se encuentra la musicoterapia) además del tratamiento convencional, buscan mejorar los efectos del mismo y ganar recursos psicológicos para hacer frente a la situación, sintiendo que la tienen "bajo control" (Downer et al., 1994).

Tomaremos como referencia un artículo recientemente publicado por Pothoulaki, MacDonald y Flowers (2006), en el que los autores realizan una revisión exhaustiva sobre intervenciones con musicoterapia llevadas a cabo en pacientes con cáncer. Este trabajo nos ofrece una visión de los estudios publicados entre 1982 y 2006 en Estados Unidos, Canadá, Australia, España, Alemania y Reino Unido. Dicha revisión consta de 37 estudios, 13 de los cuales son de naturaleza cualitativa y 10 son 
estudios de caso único. Gran parte de los trabajos revisados no controlaban variables como la edad de los participantes, el sexo, el tipo y el estado de la enfermedad, lo que hace bastante difícil realizar comparaciones fiables y saber si realmente la musicoterapia ha conseguido los objetivos esperados, ya que estas variables pueden afectar a las respuestas y comportamientos de los sujetos. Por ejemplo, no muestran las mismas actitudes los pacientes que están en fase terminal que aquellos a los que se les acaba de diagnosticar el cáncer.

La mayoría de los estudios seleccionados para esta revisión son de naturaleza cuantitativa, y utilizan un diseño entre-sujetos para poder, así, comparar los efectos que producen las sesiones de música. Cabe señalar que cinco de los estudios son diseños aleatorios. Usar este tipo de diseños implica contar con el suficiente número de pacientes para poder afirmar que las diferencias encontradas en los resultados no se deben a diferencias preexistentes entre los pacientes que formaban los diferentes grupos. En musicoterapia utilizar estudios de asignación aleatoria es bastante complicado (esto podría explicarse por razones prácticas, como la dificultad de reclutar sujetos, dados los requisitos específicos que deben cumplir), de ahí su escaso número y el hecho de que los autores afirmen que lo más adecuado en estos casos es usar diseños entre-sujetos y estudios cuasi-experimentales con un grupo control (Pothoulaki et al., 2006).

Aunque en la revisión realizada por Pothoulaki et al. (2006), se encuentran evidencias empíricas para afirmar que la intervención con musicoterapia en pacientes oncológicos produce efectos beneficiosos en conjunto, el número de estudios existentes no es suficiente para mantener dicha afirmación. Los autores mencionan cómo en este punto tan sólo se puede afirmar que existen pequeños indicios, pero que la investigación científica basada en este tema debe ser de mayor calidad, adecuándose a los parámetros científicos establecidos. Los investigadores deberían plantear su investigación antes de llevarla a cabo, establecer grupos de control adecuados para poder realizar las comparaciones oportunas $y$, por supuesto, velar para que en sus estudios se cumpla la validez interna y externa.

Recientemente, Daykin, Bunt y McClean (2006) publican un interesante estudio en el que se plantean conocer el papel que juega la musicoterapia en el sistema británico de salud. Para ello elaboran un cuestionario que mandan por vía postal a 80 responsables de organizaciones de cuidados oncológicos. Los autores realizaron tanto un análisis cuantitativo de los datos como un análisis cualitativo de los mismos. El cuestionario fue contestado y devuelto por el $84 \%$ de los encuestados (67 cuestionarios). En dicho cuestionario se preguntó si utilizaban la musicoterapia, qué tipo de actividades en concreto realizaban, si aplicaban otras terapias creativas además de la musicoterapia (por ejemplo, arteterapia), si la musicoterapia era impartida por un musicoterapeuta colegiado, con qué frecuencia realizaban las sesiones de 
musicoterapia, el formato que tenían las mismas (individual, en grupo, ambas) y de dónde obtenían los fondos para poder realizarlas. La información recabada señala que: al menos dos tercios de los encuestados utilizan más de un tipo de actividad musical, siendo la actividad más común la de escuchar música grabada; el 33\% de la muestra usa la musicoterapia junto con una o más terapias creativas (la preferida es la arteterapia -24\%-); el 24\% de los musicoterapeutas están colegiados frente a un $48 \%$ que no lo está; la frecuencia de las sesiones suele ser semanal (semanal, 55\%; mensual, $25 \%$; diaria, 29\%) siendo el formato de las sesiones tanto individual como en grupo (individuales, $14 \%$; en grupo, 28\%; ambas, $58 \%$ ); y, el $35 \%$ depende de subvenciones externas, el $20 \%$ tiene más de una fuente de financiación y el $15 \%$ funciona con fondos internos.

Por último, nos gustaría referirnos a un estudio realizado por Smolen, Topp y Singer (2002) con pacientes que son sometidos a una colonoscopia. La realización rutinaria de esta prueba se asocia con una reducción en la mortalidad del cáncer de colón (Macrae, Tan y Williams, 1983). Sin embargo, las molestias y la ansiedad que suelen acompañar a la realización de la misma son las principales razones por las cuales los pacientes rechazan someterse a dicha prueba (Palakanis, DeNobile, Sweeney y Blankenship, 1994). Por esta razón, Smolen et al. (2002) deciden explorar los efectos que la musicoterapia tiene sobre los signos de ansiedad de un grupo de treinta y dos pacientes sometidos a una colonoscopia. Los participantes en el estudio fueron asignados aleatoriamente a uno de los siguientes dos grupos: (1) experimental, escuchaban música que podían seleccionar de un repertorio de música rock, jazz, clásica y ligera, a lo largo de toda la prueba, y (2) control, se les aplicó el procedimiento estándar (sin música). Antes y después de ser sometidos a la prueba los sujetos rellenaban un cuestionario que registraba ansiedad de estado (State Anxiety Inventory (SAI, Spielberger, 1983). Además, a lo largo de todo el proceso se registraron en cuatro ocasiones signos fisiológicos de ansiedad (tasa cardiaca y presión sanguínea). Los resultados mostraron que tanto la tasa cardiaca como la presión sistólica y diastólica fueron disminuyendo a lo largo de la prueba en el grupo experimental mientras que se mantuvo constante en el grupo control. También observaron que el grupo con el que se utilizó la musicoterapia tendía a mostrar una menor ansiedad de estado, aunque esta tendencia no fue estadísticamente significativa. Finalmente, durante el desarrollo de la prueba el grupo experimental necesitó que se le administrase una menor dosis de sedante que el grupo control. Estos datos llevan a los autores a concluir que la audición de música es una técnica útil que puede ayudar a disminuir la cantidad de sedantes necesaria así como la ansiedad que muestran los pacientes que son sometidos a una colonoscopia. 


\section{Musicoterapia y enfermedades coronarias}

Dentro de este epígrafe seguiremos la división realizada por Hanser y Mandel (2005) sobre musicoterapia y enfermedades de corazón. Para una mayor comprensión de los artículos comentados dividiremos los trabajos en función de la enfermedad cardíaca sufrida por los pacientes, que en este caso será: infarto, bypass, unidades de cuidados cardíacos y pediatría cardíaca.

\section{Infarto agudo de miocardio}

En 1999, White (1999) observó cómo pacientes que habían sufrido un infarto agudo de miocardio reducen su tasa de respiración y pulsaciones después de haber escuchado veinte minutos de música. En dicho estudio se comprobó que los resultados del grupo experimental (pacientes con infarto) diferían significativamente de los efectos encontrados en el grupo control. Incluso una hora después de haber escuchado la sesión de música, los pacientes cardíacos continuaban con los efectos beneficiosos que les había provocado la sesión de musicoterapia. Estos resultados son similares a los encontrados por el mismo autor en un estudio anterior publicado en 1992.

En el estudio publicado por Bolwerk (1990), pacientes que habían sufrido un infarto agudo de miocardio escuchaban durante 22 minutos 3 temas de música clásica de Bach, Beethoven y Debussy. Las sesiones de musicoterapia tuvieron una frecuencia diaria durante el tiempo que duró la hospitalización de los pacientes (tres días). Al realizar comparaciones con un grupo control, se encontró que aquellos que habían escuchado la música informaban tener un nivel de ansiedad significativamente menor que aquellos que no habían tenido esta experiencia.

Por otro lado, Guzzetta (1989) realiza una investigación con sujetos que han sufrido un infarto de miocardio, en la que combina la musicoterapia con ejercicios de relajación. El estudio incluye tres grupos, dos experimentales y uno control. En el grupo experimental música, los pacientes realizaban los ejercicios de relajación y, después, escuchaban durante 20 minutos un cassette, de entre tres posibles, proporcionado por el experimentador. En el grupo experimental de relajación, los pacientes solamente realizaban los ejercicios de relajación. Estos dos grupos fueron comparados con un grupo control en medidas de estrés, ansiedad, tasa cardíaca y complicaciones cardíacas. El autor encuentra que los dos grupos experimentales presentan mejoras significativas en comparación al grupo control.

En otro estudio realizado por Lewin, Thompson y Elton (2002), se explora si el hecho de que el sujeto elija la música que escucha durante la sesión de musicoterapia, en función de que represente algo importante en su vida, o, por el contrario, escucha aquella que le proporciona el musicoterapeuta, influye en los 
resultados obtenidos. Curiosamente, los autores no encontraron diferencias entre ambos grupos en ninguna de las medidas registradas (de ansiedad y de calidad de vida).

\section{Bypass coronarios}

En 1996, Zimmerman, Neiveen, Barnason y Schmaderer (1996) realizaron un estudio con pacientes que habían sufrido una operación de bypass coronario. Dividieron a los pacientes en tres grupos: en la primera condición los pacientes escuchaban una música, elegida por ellos entre 5 disponibles; en la segunda condición los pacientes veían durante 30 minutos escenas relajantes acompañadas de música; y, en la tercera condición disfrutaron de 30 minutos de descanso sin interrupción. El grupo de pacientes en la condición de escucha de música sufrió menos dolor que el resto de los grupos. Además, el grupo de pacientes que vio las imágenes acompañadas de música mejoró su calidad de sueño en comparación con el grupo control. En otro estudio similar, Barnason, Neiveen y Zimmerman (1995) encontraron cambios significativos en el humor de los pacientes en la condición de escucha de música, aunque se produjo una respuesta generalizada de relajación en los tres grupos.

Por otra parte, Tusek, Cwynar y Cosgrove (1999) investigaron los efectos que tiene la visión de una serie de imágenes acompañadas de música en un conjunto de pacientes cardíacos que iban a ser operados. Los pacientes veían y escuchaban las imágenes dos veces antes de la operación y otras dos veces después de la operación (las sesiones de musicoterapia se llevaban a cabo durante la anestesia y en la sala de recuperación tras la operación). Los resultados mostraron que el tiempo de estancia en el hospital de este grupo fue significativamente menor que el de un grupo control que no recibió las sesiones de musicoterapia con imágenes.

Cabe señalar, por último, que en 1996 Standley realizó un meta-análisis donde encontró, como resultado general, que las sesiones de musicoterapia eran más eficaces en pacientes con dolores crónicos que en aquellos con dolores agudos y severos.

\section{Unidades de cuidados cardíacos}

Cadigan et al. (2001) encontraron que sonidos sinfónicos acompañados de sonidos de la naturaleza eran eficaces en la reducción de la presión sanguínea, la tasa respiratoria y la angustia psicológica en pacientes cardíacos que tenían que guardar reposo. En cambio, otras investigaciones llevadas a cabo en unidades de cuidados cardíacos muestran resultados inconsistentes sobre el efecto de la musicoterapia en este tipo de pacientes.

Distintos estudios llegan a la conclusión de que la elección de la música empleada en las sesiones de musicoterapia en las unidades de cuidados cardíacos es fundamental para el buen funcionamiento de la terapia (Elliot, 1994; Zimmerman, 
Pierson y Marker, 1988). Éste puede ser el motivo por el que diversos investigadores encuentran resultados bastante dispares en los efectos producidos en los pacientes con enfermedades cardíacas (presión, respiración, ansiedad).

\section{Pediatría cardiaca}

Dadas las peculiares características de la población que forman estas unidades, el papel de los musicoterapeutas y, por ende, el de la musicoterapia, juega un papel muy importante. En las unidades de cuidados intensivos, las sesiones de musicoterapia no sólo son una forma de mejorar los síntomas físicos que sufren los pacientes, sino que son una pieza fundamental a la hora de mejorar la calidad de vida de estos niños. La musicoterapia les ofrece una distracción, un juego, una actividad atrayente en la que pueden formar parte tanto los niños como sus familiares, pero lo más importante es que les aporta normalidad a sus vidas.

La terapia musical se centra en las habilidades y capacidades de estos pacientes, no en sus incapacidades, les ofrece una nueva forma de comunicarse entre ellos, con sus familiares y con el personal médico, y una forma de liberar y expresar sus sentimientos. Dun (1995) publica un estudio en el que muestra los beneficios que aporta la musicoterapia a los niños que necesitan de cuidados cardíacos.

\section{Musicoterapia y neonatos}

Según Standley (2001), la música sola o combinada con la voz humana puede funcionar en la unidad de cuidados intensivos para neonatos reduciendo el estrés, proporcionando estimulación en un ambiente empobrecido, promoviendo el vínculo con los padres, y facilitando el desarrollo social, comunicativo y neurológico en los niños prematuros. Por ejemplo, se ha demostrado que los estímulos auditivos rítmicos y la voz de la madre tienen un efecto calmante similar sobre estos niños (Moore, Gladstone y Standley, 1994) y que la audición de música mejora sus constantes físicas (Cassidy y Standley, 1995; Collins y Kuck, 1991; Standle y Moore, 1995) y disminuye su estancia en el hospital (Caine, 1991). En 2002 Standley realiza un metaanálisis sobre el uso de la musicoterapia con niños prematuros, en el que se incluyen 10 estudios que cumplen los siguientes criterios: (1) son estudios experimentales en grupo o de caso único; (2) todos los participantes eran niños prematuros y con bajo peso que se encontraban recibiendo tratamiento en la unidad de cuidados intensivos; (3) la música fue incluida y evaluada como una variable independiente; y, (4) el informe incluía el diseño, el procedimiento y los resultados. Los datos del estudio mostraron que la música tenía un efecto beneficioso para los niños el cual se reflejaba en todas las variables registradas: tasa cardiaca, tasa respiratoria, adquisición de peso, días en el hospital, etc. Así pues, tal y como la autora señala, estos resultados por sí mismos justificarían la incorporación de la musicoterapia en los programas utilizados para el 
cuidado de los niños prematuros (Standley, 2001, ver también para una guía sobre la utilización de esta terapia en las unidades de cuidados intensivos de neonatos).

\section{Musicoterapia y operaciones quirúrgicas}

Otro campo de acción en el que la musicoterapia está teniendo mucha aceptación es en el de pacientes que van a operarse, para reducir la ansiedad o en pacientes que ya han sido operados, para reducir el dolor. En el presente apartado haremos una pequeña revisión de los artículos más recientemente publicados sobre este tema. Nos centraremos en las características de dichos estudios y veremos si han obtenido los resultados esperados.

Evans (2002) publicó una revisión de la literatura sobre este tema, en la que aborda 19 estudios realizados entre 1990 y 1999, en la que señala que, en general, los pacientes hospitalizados que reciben musicoterapia presentan una mayor reducción de la ansiedad, pero que las sesiones de musicoterapia no afectan ni a los latidos del corazón ni a la presión sanguínea.

Recientemente, Buffum et al. (2006) publican un estudio cuasiexperimental, con asignación aleatoria de los sujetos al grupo experimental y al control, en el que se plantean comprobar si una sesión de 15 minutos de audición de música (clásica, jazz, rock, country o new age) antes de la operación, en pacientes que van a someterse a una angiografía vascular, reduce la ansiedad de los mismos. En dicho estudio participan 170 sujetos; de ellos, los 85 pacientes que conforman el grupo experimental seleccionaron la música que iban a escuchar y la escucharon durante 15 minutos inmediatamente antes y después de la operación. Los experimentadores tomaron medidas de presión arterial, pulsaciones del corazón y respiración. Con el grupo control se siguió el mismo procedimiento que con el grupo experimental, a excepción de escuchar la música; en lugar de esto se les permitió esperar en silencio antes y después de la operación en el mismo lugar y durante el mismo tiempo que los sujetos experimentales. A ambos grupos, además, se les pasó el "State Anxiety Inventory". Comparando los datos pre y post intervención, los autores encontraron diferencias estadísticamente significativas en la reducción de la ansiedad en los pacientes que habían escuchado la música frente al grupo control. Con respecto a las variables fisiológicas registradas, tan sólo se encontraron diferencias estadísticamente significativas en el pulso; los pacientes que habían escuchado la música mostraban una mayor reducción del pulso que los pacientes del grupo control. Por el contrario, los autores no encontraron diferencias en las variables presión diastólica, presión sistólica y respiración. Los resultados obtenidos son similares a los encontrados por Cooke, Chaboyer e Hiratos (2005), observándose varios grados de efecto sobre la ansiedad en otros estudios que presentan sesiones de musicoterapia con una duración que oscila entre 15 y 40 minutos (Mandle et al., 1990). 
Diversas investigaciones han encontrado que los pacientes que están en cuidados intensivos y escuchan música sedante experimentan menos ansiedad que los pacientes que reciben descanso (Chlan, 1998; White, 1992). Por el contrario, otros investigadores encuentran que tanto la música sedante como el descanso reducen la ansiedad y el dolor de los pacientes de cuidados intensivos (Barnason et al., 1995; Bolwerk, 1990; Elliot, 1994; Zimmerman, et al., 1988; Zimmerman et al., 1996). Para estos últimos investigadores no está claro qué ventajas produce cada tipo de intervención (música vs. descanso).

Los posibles efectos positivos de la música y del descanso no habían sido estudiados en pacientes sometidos a una operación a corazón abierto que se encontraban en el postoperatorio, hasta que Voss et al. (2004) realizan una investigación en la que se proponen explorar esta cuestión. Las variables estudiadas en dicha investigación fueron: nivel de ansiedad informado por el paciente, y sensación de dolor y angustia provocada por el dolor durante los 30 minutos en los que son levantados de la cama y llevados a una silla de descanso (tiempo durante el cual se les administro el tratamiento experimental). En base a los estudios publicados hasta la fecha los autores esperaban encontrar que la música redujera la ansiedad y el dolor, al ser un elemento distractor que tiene la capacidad de provocar que el paciente focalice su atención en otra cosa que no sea su propio dolor (en la música, un estímulo que, por otra parte, es más placentero), y que esto estimulara y produjera la respuesta de relajación (Benson, 2000; Good et al., 1999). El diseño utilizado fue un diseño experimental con tres grupos pre-post test, participando en el estudio una muestra total de 62 pacientes provenientes de la unidad de cuidados intensivos de un hospital que fueron asignados a las tres condiciones, dos experimentales y una control, de la siguiente manera: 19 pacientes pasaron por la condición de música, 21 por la condición de descanso programado y 21 fueron asignados a la condición control (tratamiento convencional).

Los resultados encontrados señalan que los pacientes que formaron parte del grupo música y del grupo descanso, mostraron significativamente menor ansiedad, sensación de dolor y angustia provocada por el dolor en el postest frente a las medidas tomadas antes de realizar el experimento. Concretamente, al finalizar la sesión el grupo música presentaba un $72 \%$ menos de ansiedad, un $57 \%$ menos sensación de dolor y un $69 \%$ menos de angustia que el grupo control. Por otro lado, los pacientes que formaron parte del grupo música, al finalizar la sesión presentaban un 59\% menos de ansiedad, un $51 \%$ menos sensación de dolor y un $60 \%$ menos angustia que el grupo descanso. Entre el grupo descanso y el grupo control no se encontraron diferencias estadísticamente significativas. Estos resultados están en la misma línea que los obtenidos por otros autores en estudios similares (Bolwerk, 1990; Chlan, 1998; White, 1992, 1999). 
Por otra parte, MacDonald et al. (2003), publicaron un artículo en el que presentaban un trabajo cuyo objetivo era comprobar los efectos que produce escuchar música elegida por el paciente sobre el dolor que éste siente después de una operación. En un primer estudio participaron 40 pacientes ( 23 en el grupo control y 17 en el grupo experimental) a los que se les practicó una operación menor en el pie. Los sujetos que participaron en el grupo experimental eligieron la música que iban a escuchar. La medida pre-test (utilizan dos escalas de dolor y de ansiedad) fue tomada el mismo día de la operación, la primera medida post-test fue tomada entre una hora y dos horas después de la operación (en función de las diferentes reacciones de los pacientes a la anestesia general) y la tercera medida fue registrada cuatro horas después de la operación. Los pacientes del grupo experimental escucharon la música al menos 45 minutos durante las cuatro horas del postoperatorio. Los autores encontraron que los sujetos que participaron en la condición experimental, veían disminuida su sensación de ansiedad significativamente; por el contrario, en los sujetos del grupo control la variable ansiedad no se veía alterada. En las dos medidas de dolor, los autores no encontraron que éste se redujese en el grupo experimental.

A la vista de estos resultados se realizó un segundo estudio, con la misma base y procedimiento del estudio previo, aunque se incluyeron las siguientes modificaciones: seleccionaron mujeres (30 en el grupo experimental y 28 en el grupo control) que iban a ser sometidas a una histerectomía (la operación es más grave y provoca mayor dolor al paciente), cambiaron uno de los test que medía dolor $\mathrm{y}$, finalmente, ampliaron el intervalo de intervención postoperatorio de 4 a 72 horas. En este estudio los autores no encontraron diferencias estadísticamente significativas entre los grupos en ninguna de las tres medidas en los tres momentos en las que fueron tomadas. En resumen, no se observó que las sesiones de música disminuyesen el dolor de los pacientes, independientemente de que la operación que se les realizase fuera de mayor o menor gravedad. Lo que sí se encontró es que en la operación de pie reducían el nivel de ansiedad mostrado por los pacientes sometidos a la misma.

\section{CONCLUSIONES}

De la revisión que acabamos de realizar se desprende que hay indicios que señalan que la musicoterapia parece ser beneficiosa para paliar los déficit emocionales, atencionales, motores, de comunicación, etc., asociados con distintas patologías, y para reducir la ansiedad que puede aparecer en situaciones concretas (v.g., por enfermedad o ante una operación). Sin embargo, hay que señalar que la mayor parte de los datos disponibles se derivan o de estudios de caso único o de estudios de grupo que presentan serios problemas metodológicos (v.g., ausencia de grupo control). Generalmente los terapeutas que utilizan la terapia musical han argumentado que sólo pueden realizar 
estudios de caso único ya que los programas de entrenamiento deben ser individualizados y no se puede utilizar la misma terapia para varios pacientes. Sin embargo, como Accordino et al. (2007) señalan, la musicoterapia no debe ser diferente de cualquier otra área de investigación científica. Los estudios deben contar con grupos control, se han de realizar diseños en los que los sujetos se asignen aleatoriamente a las diferentes condiciones experimentales, los datos deben ser analizados estadísticamente, etc.

A rasgos generales, los resultados obtenidos hasta el momento parecen indicar que la musicoterapia provoca efectos relajantes en los pacientes, lo que podría hacer que mejoraran sus síntomas físicos, aunque aún no se han encontrado datos que apoyen que las sesiones de musicoterapia alivian el dolor que éstos padecen. Queremos hacer hincapié en el hecho de que la literatura publicada sobre este tema no es lo suficientemente amplia como para realizar afirmaciones categóricas sobre los efectos que la terapia musical produce. Los profesionales que trabajan en este campo deberían tomar conciencia de la necesidad de llevar a cabo experimentos controlados con los que demostrar científicamente si esta terapia es eficaz, y ver en qué medida se producen y qué condiciones son las más adecuadas y necesarias para que se puedan observar efectos beneficiosos de la musicoterapia sobre la salud. La realización de estos estudios, y su posterior publicación en medios especializados, significaría un gran avance y una ayuda para otros profesionales del campo, ya que no partirían desde cero en sus investigaciones o en sus sesiones de musicoterapia. Además, tratándose de una técnica con la que podemos ayudar a reducir síntomas físicos negativos, tenemos que ser mucho más meticulosos y precavidos a la hora de hacer afirmaciones sobre los efectos de la misma, sobre todo, para no crear falsas expectativas. Si la musicoterapia puede ayudar de alguna forma a los pacientes hospitalizados, tal y como parece desprenderse de los estudios que señalan, al menos, una reducción en la ansiedad de los mismos, todos debemos unir nuestras fuerzas y ayudar en lo posible para que se realicen estudios experimentales adecuados y para que el conocimiento científico se difunda, sirviendo de base, a los musicoterapeutas, que cada día intentan mejorar la calidad de vida de estas personas.

\section{Agradecimientos}

La realización de este trabajo ha sido posible gracias a los proyectos SEJ2006-14723, SEJ2007-60217 y CSD2008-00048 financiados por la Dirección General de Investigación del Ministerio de Ciencia e Innovación, y SEJ-03036, financiado por la Junta de Andalucía. 


\section{REFERENCIAS}

Accordino, R., Comer, R. \& Heller, W.B. (2007). Searching for music's potential: A critical examination of research on music therapy with individuals with autism. Research in Autism Spectrum Disorders, 1, 101-115.

Aldridge, D. (2003). Music therapy references relating to cancer and palliative care. British Journal of Music Therapy, 17(1), 17-25.

Alvin, J. (1978). Musicoterapia. Buenos Aires: Ed. Ricordi.

Applebaum, E., Egel, A., Koegel, R. \& Imhoff, B. (1979). Measuring musical abilities of autistic children. Journal of Autism and Developmental Disorders, 9, 279-285.

Barnason, S., Neiveen, J. \& Zimmerman, L. (1995). The effects of music interventions on anxiety in the patient after coronary artery bypas grafting. Heart Lung, 24, 124-132.

Benenzon, R. (1981). Manual de Musicoterapia. Barcelona: Paidós.

Benson, H. (2000). The relaxation response. New York: HarperTorch.

Bolton, A. \& Adams, M. (1983). An investigation of the effects of music therapy on a group of profoundly mentally handicapped adults. International Journal of Rehabilitation Research, 6(4), 51 1-512.

Bolwerk, C.L. (1990). Effects of relaxing music on state anxiety in myocardial infarction patients. Critical Care Nursing Quarterly, 13, 63-72.

Bonnel, A., Mottron, L., Peretz, I., Trudel, M., Gallun, E. \& Bonnel, A.M. (2003). Enhanced pitch sensitivity in individuals with autism: A signal detection analysis. Journal of Cognitive Neuroscience, 15, 226-235.

Buffum, M.D., Sasso, C., Sands, L.P., Lanier, E., Yellen, M. \& Hayer, A. (2006). A music intervention to reduce anxiety before vascular angiography procedures. Journal of Vascular Nursing, 24(3), 68-73.

Cadigan, M.E., Caruso, N.A., Haldeman, S.M., McNamara, M.E., Noyes, D.A., Spadafora, M.A. \& Carroll, D.L. (2001). The effects of music on cardiac patients on bed rest. Progress in Cardiovascular Nursing, 16, 5-13.

Caine, J. (1991). The effects of music on the selected stress behaviors, weight, caloric and formula intake, and length of hospital stay of premature and low birth weight neonates in a newborn intensive care unit. Journal of Music Therapy, 28, 180-192.

Cassidy, J.W. \& Standley, J.M. (1995). The effect of music listening on physiological responses of premature infants in the NICU. Journal of Music Therapy, 32, 208-227.

Chlan, L. (1998). Effectiveness of a music therapy intervention on relaxation and anxiety for patients receiving ventilatory assistance. Heart Lung, 27, 169-176.

Cohen, N.S. \& Masse, R. (1993). The application of singing and rhythmic instruction as a therapeutic intervention for persons with neurogenic communication disorders. Journal of Music Therapy, 30, 81-99.

Coleman, S. (2002). Music therapy for learners with severe disabilities in a public school setting. In B. Wilson (Ed.), Models of music therapy interventions in school settings (2nd ed., pp. 197-209). Silver Spring, MD:The American Music Therapy Association, Inc.

Collins, S.K. \& Kuck, K. (1991). Music therapy in the neonatal intensive care unit. Neonatal Network, 9(6), 23-26.

Cooke, M., Chaboyer, W. \& Hiratos, M.A. (2005). Music and its effect on anxiety in short waiting periods: a critical appraisal. Journal of Clinical Nursing, 14, 145-155.

Daykin, N., Bunt, L. \& McClean, S. (2006). Music and healing in cancer care: A survey of supportive care providers. The Arts in Psychotherapy, 33, 402-413. 
DiGiammarino, M. (1990). Functional music skills of persons with mental retardation. Journal of Music Therapy, 27, 209-220.

Dorow, L.G. \& Horton, J.J. (1982). Effect of the proximity of auditory stimuli and sung versus spoken stimuli on activity levels of severely/profoundly mentally retarded females. Journal of Music Therapy, 19, 114-124.

Downer, S.M., Cody, M.M., McCluster, P., Wilson, P.D., Arnott, S.J., Lister, T.A. \& Slevin, M.L. (1994). Pursuit and practice of complementary therapies by cancer patients receiving conventional treatment. British Medical Journal, 309, 86-89.

Dun, B. (1995). A different beat: music therapy in children's cardiac care. Music Therapy Perspectives, 13, 35-39.

Elliot, D. (1994). The effects of music and muscle relaxation on patient anxiety in a coronary care unit. Heart Lung, 23, 27-35.

Evans, D. (2002). The effectiveness of music as an intervention for hospital patients: a systematic review. Journal of Advanced Nursing, 337, 8-18.

Foxton, J.M., Stewart, M.E., Barnard, L., Rodgers, J., Young, A. H., O’Brien, G. y Griffiths, T.D. (2003). Absence of auditory 'global interference' in autism. Brain, 126, 27032709.

Gaston, E.T. (1957). Factors contributing to responses to music. In K. Lawrence (Ed.), Book of Proceedings (pp. 23). Silver Spring, MD: National Association for Music Therapy.

Gaston, E.T. (1968). Tratado de Musicoterapia. Buenos Aires: Paidós.

Gibbons, A.C. (1983). Rhythm responses in emotionally disturbed children with differing needs for external structure. Music Therapy, 3(1), 94-102.

Gladfelter, N. (2002). Music therapy for learners with learning disabilities in a private day school. In B. Wilson (Ed.), Models of music therapy interventions in school settings (2nd ed., pp. 278-290). Silver Spring, MD: The American Music Therapy Association, Inc.

Good, M., Stanton-Hicks, M., Grass, J.A., Cranston Anderson, G., Choi, C., Schoolmeesters, L.J. \& Salman, A. (1999). Relief of postoperative pain with jaw relaxation, music and their combination. Pain, 81, 163-172.

Gunsberg, A. (1988). Improvised musical play: A strategy for fostering social play between developmentally delayed and nondelayed preschool children. Journal of Music Therapy, 25, 178-191.

Guzzetta, C.E. (1989). Effects of relaxation and music therapy on patients in a coronary unit with presumptive acute myocardial infarction. Heart Lung, 18, 609-616.

Hanks, S. (1986). The role of therapy in Rett syndrome. American Journal of Medical Genetics Supplement, 1, 247-252.

Hanser, S.B. (2005). Challenges of music therapy in a world of need. Arts in Psychotherapy, 32, 217-224.

Hanser, S.B. y Mandel, S.E.(2005). The effect of music therapy in cardiac healthcare. Cardiology in review, 13(1), 18-23.

Heaton, P. (2003). Pitch memory, labeling, and disembedding in autism. Journal of Child Psychology and Psychiatry, 44, 543-551.

Heaton, P. (2004). Interval and contour processing in autism. Journal of Autism and Developmental Disorders, 35(6), 787-793.

Heaton, P., Hermelin, B. \& Pring, L. (1998). Autism and pitch processing: A precursor for savant musical ability? Music Perception, 15, 291-305.

Heaton, P., Pring, L. \& Hermelin, B. (2001). Musical processing in high functioning children with autism. Annals of the New York Academy of Sciences, 930, 443-444. 
Hillecke, T., Nickel, A. \& Bolay, H.V. (2005). Scientific perspectives on music therapy. Annals of the New York Academy of Sciences, 1060, 271-282.

Howell, R.D., Flowers, P.J. \& Wheaton, J.E. (1995). The effects of keyboard experiences on rhythmic responses of elementary school children with physical disabilities. Journal of Music Therapy, 32, 91-112.

Hughes, J., Robbins, B., McKenzie, B. \& Robb, S. (1990). Integrating exceptional and nonexceptional young children through music play: A pilot program. Music Therapy Perspectives, 8, 52-56.

Humpal, M. (1991). The effects of an integrated early childhood music program on social interaction among children with handicaps and their typical peers. Journal of Music Therapy, 28(3), 161-177.

Juslin, P.N. \& Sloboda, J.A. (2001). Music and emotion, theory and research. New York: Oxford University Press.

Knill, C. (1983). Body awareness, communication and development: A programme employing music with the profoundly handicapped. International Journal of Rehabilitation Research, 6(4), 489-492.

Kruse, J. (2003). Music therapy in United States cancer settings: Recent trends in practice. Music Therapy Perspectives, 21, 89-98.

Lacarcel, M.J. (1990). Musicoterapia en Educación Especial. Murcia: Servicio de Publicaciones de la Universidad de Murcia.

Lewin, R.J., Thompson, D.R. \& Elton, R.A. (2002). Trial of the effects of an advice and relaxation tape given within the first 24 hours of admission to hospital with acute myocardial infarction. International Journal of Cardiology, 82, 107-114.

MacDonald, R.A.R., Mitchell, L.A., Dillon, T., Serpell, M.G., Davies, J.B. y Ashley, E.A. (2003). An empirical investigation of the anxiolytic and pain reducing effects of music. Psychology of Music, 31(2), 187-203.

Macrae, F.A., Tan, K.G. y Williams, C.B. (1983). Towards safer colonoscopy: a report on the complications of 5000 diagnostic or therapeutic colonoscopies. Gut, 24(5), 376-383.

Madsen, C.K. (1981). Music therapy: A behavioural guide for the mentally retarded. Washington, DC: National Association for Music Therapy.

Mandle, C.L., Domar, A.D., Harrington, D.P., Leserman, J., Bozadjian, E.M., Friedman, R. \& Benson, H. (1990). Relaxation response in femoral angiography. Radiology, 174(3), 737-739.

Moore, R., Gladstone, I. \& Standley, J. (1994). Effects of music, maternal voice, intrauterine sounds and white noise on the oxygen saturation levels of premature infants. Trabajo presentado en the Annual Conference, National Association for Music Therapy, Inc, Orlando.

Moore, R. \& Mathenius, L. (1987). The effects of modelling, reinforcement, and tempo on imitative rhythmic responses of moderately retarded adolescents. Journal of Music Therapy, 24, 160-169.

Mottron, L., Peretz, I. \& Menard, E. (2000). Local and global processing of music in highfunctioning persons with autism: Beyond central coherence? Journal of Child Psychology and Psychiatry, 41, 1057-1065.

Nordoff, P. \& Robbins, C. (1977). Creative Music Therapy. New York: John Day.

Oldfield, A. \& Adams, M. (1990). The effects of music therapy on a group of profoundly mentally handicapped adults. Journal of Mental Deficiency Research, 34(2), 107-125

Palakanis, K.C., DeNobile, J.W., Sweeney, W.B. \& Blankenship, C.L. (1994). Effect of music therapy on state anxiety in patients undergoing flexible sigmoidoscopy. Diseases of the Colon and Rectum, 37, 478-481. 
Poch, S. (2002). Compendio de Musicoterapia. Volumen I. Barcelona: Herder.

Popovici, M. (1995). Melodic intonation therapy in the verbal decoding of aphasics. Romanian Journal of Neurology and Psychiatry, 33, 57-97.

Pothoulaki, M., MacDonald, R. \& Flowers, P. (2006). Methodological issues in music interventions in oncology settings: A systematic literature review. The Arts in Psychotherapy, 33, 446-455.

Preza, B., Baboci, H., Ashta, A. \& Lleshi, L. (1990). The Rett syndrome-Clinical presentation of the first 9 Albanian cases. Brain \& Development, 12(1), 40-43.

Rejto, A. (1973). Music as and aid in the remediation of learning disabilities. Journal of Learning Disabilities, 6, 286-295.

Rickson, D.J. (2006). Instructional and improvisitional models of music therapy with adolescents who have attention deficit hyperactivity disorder (ADHD): A comparison of the effects on motor impulsivity. Journal of Music Therapy, 43(1), 39-62.

Ruiz, Y.M. (2005). La musicoterapia en la educación especial. Alonso Cano. Revista Andaluza de Arte, 6. En http://perso.wanadoo.es/alonsocano1601/anteriores.htm

Sausser, S. \& Waller, R.J. (2006). A model for music therapy with students with emotional and behavioral disorders. The Arts in Psychotherapy, 33, 1-10.

Seybold, C. (1971). The value and use of music activities in the treatment of speech delayed children. Journal of Music Therapy, 8(2), 102-110.

Smolen, D., Topp, R. \& Singer, L. (2002). The effect of self-selected music during colonoscopy on anxiety, heart rate, and blood pressure. Applied Nursing Research, 16(2), 126-136.

Spielberger, C.D. (1983). Manual for the state-trait anxiety inventory (Form Y). Palo Alto: Consulting Psychologists Press, Inc.

Standley, J.M. (1996). Music research in medical/dental treatment: an update of a prior metaanalysis. In C.E. Furman (Ed.), Effectiveness of Music Therapy Procedures: Documentation of Research and Clinical Practice (pp. 1-60). Silver Spring, MD: National Association for Music Therapy.

Standley, J.M. (2001). Music therapy for the neonate. Newborn and Infant Nursing Reviews, l(4), 211-216.

Standley, J.M. (2002). A meta-analysis of the efficacy of music therapy for premature infants. Journal of Pediatric Nursing, 17(2), 107-113.

Standley, J.M. y Moore, R. (1995). Therapeutic effects of music and mother's voice on premature infants. Pediatric Nursing, 21(6), 509-512.

Taylor, D. (1990). Childhood sequential development of rhythm, melody and pitch. In F. Wilson y F. Roehmann (Eds.), Music \& Child Development (pp. 241-253). St Louis: MMB Music, Inc.

Thau, M.H. (1988). Rhythmic intervention techniques in music therapy with gross motor dysfunctions. The Arts in Psychotherapy, 15, 127-137.

Thaut, M. (1985). The use of auditory thythm and rhythmic speech to aid temporal muscular control in children with gross motor dysfunction. Journal of Music Therapy, 22, 108128.

Thaut, M. (1992). Music therapy in the rehabilitation of stroke and traumatic brain-injured clients. In W.B. Davis, K.E. Gfeller \& M.H. Thaut (Eds.), An Introduction to Music Therapy Theory and Practice (pp. 251-273). Dubuque, IA: Wm. C. Brown.

Tusek, D.L., Cwynar, R. \& Cosgrove, D.M. (1999). Effect of guided imager on lenght of stay, pain and anxiety in cardiac surgery patients. Journal of Cardiovascular Manager, 20, $22-28$. 
Voss, J.A., Good, M., Yates, B., Baun, M.M., Thompson, A. \& Hertzog, M. (2004). Sedative music reduces anxiety and pain during chair rest after open-heart surgery. Pain, 112, 197-203.

Wesecky, A. (1986). Music therapy for children with Rett syndrome. American Journal of Medical Genetics Supplement, 1, 253-257.

White, J.M. (1992). Music therapy: an intervention to reduce anxiety in the myocardial infarction patient. Clinical Nurse Specialist, 6, 58-63.

White, J.M. (1999). Effects of relazing music on cardiac autonomic balance and anxiety after acute myocardiar infarction. American Journal of Critical Care, 8, 220-227.

Wigram, T., Pederson, I.N. \& Bond, L.O. (2002). A comprehensive guide to music therapy. London: Jessica Kingsley Publishers.

Yasuhara, A. \& Sugiyama, Y. (2001). Music therapy for children with Rett syndrome. Brain and Development, 23(1), 582-584.

Zappella, M. (1986). Motivational conflicts in Rett syndrome. American Journal of Medical Genetics Supplement, 1, 143-151.

Zimmerman, L., Pierson, M.A. \& Marker, J. (1988). Effects of music on patient anxiety in coronary care units. Heart Lung, 17, 560-566.

Zimmerman, L., Neiveen, J., Barnason, S. \& Schmaderer, M. (1996). The effects of music interventions on postoperative pain and sleep in coronary artery bypass graft (GABG) patients. Scholarly Inquiring for Nursing Practice, 10, 153-169.

Recibido: 29 de octubre de 2008 Modificaciones: 23 de diciembre de 2008

Aceptado: 15 de enero de 2009 